\title{
Inflammatory Mechanisms of Organ Crosstalk during Ischemic Acute Kidney Injury
}

\author{
Laura E. White ${ }^{1}$ and Heitham T. Hassoun ${ }^{1,2}$ \\ ${ }^{1}$ Department of Surgery, The Methodist Hospital and Research Institute, Houston, TX 77030, USA \\ ${ }^{2}$ The Methodist DeBakey Heart \& Vascular Center, Houston, TX 77030, USA
}

Correspondence should be addressed to Heitham T. Hassoun, hhassoun@tmhs.org

Received 15 January 2011; Accepted 10 March 2011

Academic Editor: Niels Olsen Saraiva Camara

Copyright (C 2012 L. E. White and H. T. Hassoun. This is an open access article distributed under the Creative Commons Attribution License, which permits unrestricted use, distribution, and reproduction in any medium, provided the original work is properly cited.

\begin{abstract}
Acute kidney injury (AKI) is a common complication during inpatient hospitalization, and clinical outcomes remain poor despite advancements in renal replacement therapy. AKI in the setting of multiple organ failure (MOF) remains a formidable challenge to clinicians and incurs an unacceptably high mortality rate. Kidney ischemia-reperfusion injury (IRI) incites a proinflammatory cascade and releases cellular and soluble mediators with systemic implications for remote organ injury. Evidence from preclinical models cites mechanisms of organ crosstalk during ischemic AKI including the expression of cellular adhesion molecules, lymphocyte trafficking, release of proinflammatory cytokines and chemokines, and modification of the host innate and adaptive immune response systems. In this paper, the influence of kidney IRI on systemic inflammation and distant organ injury will be examined. Recent experimental data and evolving concepts of organ crosstalk during ischemic AKI will also be discussed in detail.
\end{abstract}

\section{Introduction}

Despite advancements in renal replacement therapy, acute kidney injury (AKI) is a frequent complication with severe implications for the critically ill patient. Published mortality rates for intensive care unit patients with AKI range between 30 and $70 \%$, and AKI alone remains an independent risk factor for mortality even after adjustment for demographics and severity of illness $[1,2]$. Kidney ischemia-reperfusion injury (IRI) occurs in various clinical settings including shock, sepsis, organ transplantation, and vascular surgery. AKI, however, rarely occurs in isolation. It has become apparent that clinically much of the high patient mortality can be attributed to the onset of systemic inflammatory response syndrome (SIRS) and progression to multiple organ failure (MOF).

Since the 1950s, clinicians have identified abnormal chest radiographs in patients with chronic kidney failure. Pulmonary dysfunction was thought to result from "increased permeability of congested pulmonary capillaries," and this was dubbed the "uremic lung" [3]. Even in the acute setting, however, kidney dysfunction directly contributes to the onset of remote organ injury. For example, increased kidney ischemia time during complex aortic surgery is associated not only with acute and chronic renal failure, but also with an increased incidence of remote organ injury and death [46].

Clinical and translational laboratory studies have demonstrated the relevance of interactions between the injured kidney and distant organs, and complex mechanisms of crosstalk between injured kidneys and remote organs such as the lungs, liver, heart, gut, brain, and hematologic system have been identified. Recent data highlights the importance of both the innate and adaptive immune response, activation of proinflammatory cascades, and an alteration of transcriptional events in remote organs during ischemic AKI. The purpose of this paper is to review emerging concepts of organ crosstalk and recent experimental data regarding the activation and systemic expression of proinflammatory pathways during ischemic AKI. For a complete list of abbreviations used in this paper, please see Table 1. 
TABLE 1: Abbreviations.

\begin{tabular}{ll}
\hline$\alpha$-MSH & $\alpha$-Melanocyte-stimulating hormone \\
AKI & Acute kidney injury \\
ALI & Acute lung injury \\
AP-1 & Activator protein-1 \\
ARDS & Acute respiratory distress syndrome \\
CNS & Central nervous system \\
G-CSF & Granulocyte colony-stimulating factor \\
GFAP & Glial fibrillary acidic protein \\
HSC & Hepatic stellate cell \\
ICAM-1 & Intercellular adhesion molecule-1 \\
IL-1 & Interleukin-1 \\
Il1r2 & Interleukin-1 receptor type II \\
IRI & Ischemia-reperfusion injury \\
KC & Keratinocyte chemoattractant \\
KC/CXCL1 & Keratinocyte-derived chemokine \\
MIP-2/CXCL-2 & Macrophage inflammatory protein \\
MOF & Multiple organ failure \\
NF- $\kappa$ B & Nuclear factor- $\kappa$ B \\
Ngp & Neutrophilic granule protein \\
Saa3 & Serum amyloid A3 \\
SIRS & Systemic inflammatory response syndrome \\
TCR & T-cell receptor \\
TLR & Toll-like receptor \\
TNFR1 & Tumor necrosis factor receptor-1 \\
TNF $\alpha$ &
\end{tabular}

\section{Ischemic AKI}

Kidney IRI remains a major cause of AKI in both native and transplanted organs and lacks a specific treatment aside from renal replacement therapy. The local effects of kidney IRI begin in the most vulnerable regions of the organ, after which a cascade of microvascular inflammation propagates. Although $25 \%$ of cardiac output contributes to renal blood flow, only a fraction ultimately reaches the vasa recta of the renal medulla, with the majority reaching the renal cortex [7]. Therefore, even slight alterations in total renal blood flow may lead to anoxic injury in the medulla, resulting in tubular dysfunction, salt wasting, and glomerular vasoconstriction through tubuloglomerular feedback $[8,9]$.

Kidney IRI activates an inflammatory response which results in endothelial cell activation, leukocyte adhesion and entrapment, and compromised microvascular blood flow [10]. Inflammation in the postischemic kidney triggers the upregulation of leukocyte adhesion molecules, toll-like receptors, and downstream transcription factors, which all contribute to disruption of the integrity of the renal vascular endothelium [11]. Adhesion molecules such as integrins and selectins along with proinflammatory cytokines propagate cellular injury not only locally in the renal tubular epithelial cells but also travel to remote organs where genomic markers of injury are upregulated and phenotypic injury occurs [12]. Unfortunately, selective inhibition of cytokines and adhesion molecules such as tumor necrosis factor- $\alpha$ (TNF- $\alpha$ ) and intercellular adhesion molecule (ICAM-1) have failed to demonstrate global attenuation of both local and remote organ injury during experimental models of ischemic AKI [13-16]. However, $\alpha$-melanocyte-stimulating hormone $(\alpha-$ MSH), a cytokine with broad antiinflammatory, anticytotoxic, and antiapoptotic properties, has demonstrated success in treating the inflammatory phenotype in rodents. In this model, treatment with $\alpha$-MSH has attenuated both renal and pulmonary injury during ischemic AKI $[13,17]$.

The largely unsuccessful effort to ameliorate MOF with specific anti-inflammatory therapeutics highlights the complexity of the systemic response to kidney IRI. From these early experimental studies, it is possible that the multiple inflammatory pathways activated in each organ system represent a unique response to ischemic AKI. Despite a paucity of data, several different pathophysiologic responses to ischemic AKI in remote organs have been identified (Figure 1). In the heart, increased expression of TNF $\alpha$ and interleukin-1 (IL-1) are associated with myocyte apoptosis. In the brain, increased expression of chemokines including keratinocyte chemoattractant (KC, a brain IL8 homologue) and granulocyte colony-stimulating factor (G-CSF) are seen with increased vascular permeability. Additional responses in the lungs, gut, and liver have been discovered, and these individual organ responses will be detailed later in this paper. We will now focus on cellular mediators, specifically those involved in the innate and adaptive immune system, which may connect local ischemic AKI with distant organ injury.

2.1. Innate Immunity. The innate immune system plays an important role in mediating the inflammatory response during ischemic AKI. Traditionally, innate immunity elicits an immediate, preprogrammed response to tissue injury that lacks immunologic memory. It is composed of plasma proteins (complement), cells (neutrophils, macrophages, and natural killer cells), and physical barriers. Proposed initiators of the innate immune response during IRI include the activation of toll-like receptors (TLRs) and the release of reactive oxygen species (nitric oxide and superoxide anion) and mitochondrial products [18]. The complement system, particularly the alternative pathway, is activated, stimulating release of cytokines and subsequent activation of neutrophils, endothelium, and macrophages [19].

TLRs are a family of transmembrane proteins which serve as major pattern recognition receptors, binding to a wide range of microbial products and endogenous ligands released as a consequence of injury. TLR-dependent signaling serves as a rapid response mechanism to local tissue damage and has been implicated in early activation of the innate immune response during kidney IRI $[11,20]$. Renal tubular epithelial cells constitutively express TLR2 and TLR4, and IRI results in selective upregulation of these TLRs. Experimental studies of kidney IRI have demonstrated attenuation of renal structural and functional injury in both TLR2-/- and TLR4-/-mice, implicating TLR2 and TLR4 signaling in renal damage [21, 22]. TLR2 may also play a role in transplantation tolerance 


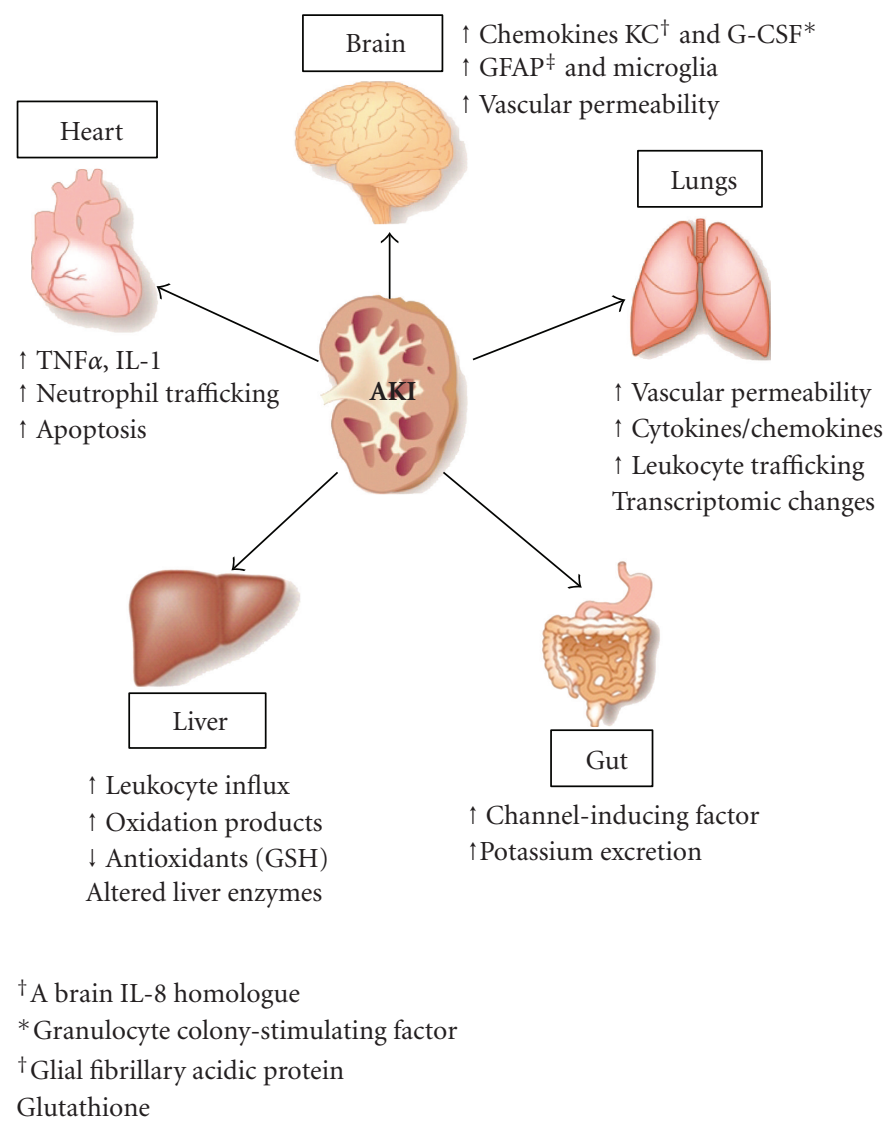

FIGURE 1: AKI and organ crosstalk. AKI induces remote organ injury in the heart, brain, lungs, liver, and gut involving multiple inflammatory pathways, including increased expression of soluble proinflammatory mediators, innate and adaptive immunity, cellular apoptosis, physiologic derangements and genomic changes.

by decreasing the infiltration of $\mathrm{T}$ cells, dendritic cells, and macrophages [23].

T cells may act during early IRI despite lacking alloantigenic stimulation, which challenges the traditional function of $\mathrm{T}$ cells as exclusive mediators of the adaptive immune response [19]. Significant evidence exists to support the role of $\mathrm{T}$ cells in gut, heart, and lung ischemia-reperfusion injury [24-28]. Likewise, experimental data also points toward antigen-independent T-cell activation in ischemic AKI through cytokines secreted by macrophages and dendritic cells, chemokines, oxygen free radicals and the complement system $[7,19,29]$.

Specific populations of T cells likely contribute to injury in unique ways. For example, mice deficient in $\mathrm{CD} 4+$ and $\mathrm{CD} 8+$ cells and athymic mice demonstrate protection from experimental ischemic AKI; however, only adoptive transfer of $\mathrm{T}$ cells of the CD4+ type restores this injury [30, 31]. Not all $\mathrm{T}$ cells, however, perpetuate inflammatory damage during ischemic AKI; some populations of $\mathrm{T}$ cells confer renal protection after IRI, particularly the $\mathrm{Th}_{2}$ phenotype of $\mathrm{CD} 4+\mathrm{T}$ cells and $\mathrm{T}$ regulatory cells [32]. Additional recent experimental data has characterized specific populations of $\mathrm{T}$ lymphocytes trafficking to remote organs that may facilitate organ crosstalk during ischemic AKI. In a rodent model of kidney IRI, a distinct influx of $\mathrm{CD} 3+(\mathrm{T})$ cells with a predominant CD8+ $\mathrm{T}$ cell subpopulation was identified 24 hours after experimental kidney IRI. These pulmonary $\mathrm{T}$ cells acquired increased expression of activation markers suggesting that ischemic AKI induced trafficking of activated $\mathrm{T}$ cells which contributed to pulmonary injury, specifically apoptosis [33]. The specific role of subpopulations of T cells in the pathogenesis of ischemic AKI remains a stimulating topic of current and future investigation, both in the innate and adaptive immune response to kidney IRI.

2.2. Adaptive Immunity. The adaptive immune system relies on the specificity of antigen receptors on both $\mathrm{B}$ and $\mathrm{T}$ cells that respond to millions of various antigenic molecular structures. Once stimulated by an antigen, B cells (humoral immunity) produce specific antibodies, perform opsonization to facilitate phagocytosis, and activate the complement system. The T-cell receptor (TCR) responds to presented antigen peptides by activating macrophages to kill phagocytosed microbes, directly destroying infected cells and by releasing cytokines to promote further response (cellmediated immunity).

Antigen-dependent T-cell activation has been demonstrated in experimental models of renal IRI $[34,35]$. For example, mice with a restricted TCR cell repertoire suffer 
less injury and promote a far diminished inflammatory response during renal IRI. Additionally, athymic mice that subsequently underwent adoptive transfer with these TCRrestricted $\mathrm{T}$ cells failed to restore injury as severely as seen with transfer of wild-type T-cells [36]. T cells also play a role in long term renal modification, as an increased number of both activated and effector-memory $\mathrm{T}$ cells have been observed in postischemic kidneys as long as 6 weeks after IRI [37]. These T cells may in fact recognize antigens released during kidney IRI and subsequently target the kidney in an autoimmune response, leading to long-term progression of renal dysfunction. This hypothesis was demonstrated in a murine adoptive transfer model in which naïve mice received $\mathrm{T}$ cells from mice who were 6 weeks after kidney IRI and subsequently developed albuminuria [38]. Little is known about the role of B cells in renal IRI; however, Bcell-deficient mice demonstrated renal protection in the early phase of experimental IRI [39].

In summary, both the innate and adaptive immune responses play critical roles in the pathophysiology of ischemic AKI. Either following antigen stimulation or in the presence of proinflammatory chemokines and oxygen free radicals, $\mathrm{T}$ cells undergo early activation and serve as a bridge between adaptive and innate immunity. This specific host immune response to kidney IRI facilitates distant organ crosstalk along with soluble proinflammatory mediators and will be further discussed in the following paragraphs.

\section{Evolving Concepts of Organ Crosstalk during Ischemic AKI}

3.1. Kidney-Lung Crosstalk. The combination of AKI with acute lung injury (ALI) remains a formidable challenge to clinicians caring for critically ill patients. In the setting of MOF, AKI and ALI occur more frequently together than any other combination of organ systems, and predicted mortality approaches $80 \%[40,41]$. This exceedingly high mortality cannot be attributed solely to volume overload; leukocyte trafficking, uremic toxins, and oxidative stress mechanisms all likely contribute to this devastating clinical syndrome. For the purposes of this paper, ALI represents a $\mathrm{P}_{\mathrm{a}} \mathrm{O}_{2} / \mathrm{FiO}_{2}$ ratio $<300$ combined with chest radiograph findings of acute bilateral infiltrates in the absence of elevated cardiac filling pressures, as defined by the America-European Consensus Conference on Acute Respiratory Distress Syndrome (ARDS) [42].

While the mechanisms for ischemic AKI-induced ALI remain incompletely understood, several studies point toward a self-propagating cycle with activation of proinflammatory and proapoptotic pathways (Figure 2). AKI leads to lung injury and inflammation, and in turn, ALI and its attendant hypoxemia and hypercapnia worsened by mechanical ventilation with high positive end-expiratory pressure leads to diminishing renal hemodynamics and function. Lung injury during ischemic AKI features marked pulmonary vascular permeability, erythrocyte sludging in lung capillaries, interstitial edema, focal alveolar hemorrhage, and inflammatory cell infiltration [12, 43, 44]. Mechanisms for decreased alveolar fluid clearance include downregulation of pulmonary epithelial salt and water transporters including $\mathrm{ENaC}, \mathrm{Na}, \mathrm{K}-\mathrm{ATPase}$, and aquaporins during ischemic AKI [43-45]. This specific response likely contributes to the increased microvascular permeability and clinical pulmonary edema frequently encountered in the setting of ischemic AKI and MOF.

3.1.1. Role of Cytokines, Chemokines, and Leukocyte Trafficking. Experimental studies have identified a distinct pulmonary functional response and genomic signature induced during ischemic AKI which differs from that induced by uremia alone. A comprehensive genomic map and ontology analysis revealed many of these top differentially expressed genes participating in proinflammatory and proapoptotic pathways. Genes with early and sustained activation at 6 and 36 hours after ischemic AKI included neutrophilic granule protein (Ngp), serum amyloid A3 (Saa3), and Interleukin 1receptor type II (Il-1r2) [12].

Further investigation by Deng et al. [13] has also identified an early pulmonary inflammatory response with rapid activation of transcription factors NF- $\kappa \mathrm{B}$ and $\mathrm{AP}-1$ during ischemic AKI. By 4 hours, lung expression of TNF$\alpha$ and ICAM-1 led to accumulation of pulmonary neutrophils. Leukocytes play a fundamental role in the development of ALI, and several studies have documented lung leukocyte activation and trafficking during experimental AKI with early and sustained neutrophil sequestration $[46,47]$. Additional experiments by Klein et al. have demonstrated early expression of the neutrophil chemokine keratinocyte-derived chemokine (KC/CXCL1) and macrophage inflammatory protein (MIP-2/CXCL-2) along with increased pulmonary myeloperoxidase activity and pulmonary microvascular permeability during ischemic AKI [45]. The authors also examined interleukin-6, a neutrophilrecruiting chemokine previously identified as a candidate gene by preferential expression in whole lung tissue during ischemic AKI [48], and determined it was critical for influencing lymphocyte trafficking and pulmonary permeability [45].

While neutrophils are key mediators in several extrapulmonary models of ALI such as sepsis and mesenteric IRI, their role in ischemic AKI-induced ALI remains to be elucidated. Uremic neutrophils have even demonstrated a protective effect in the setting of ALI [49]. Clearly, leukocyte trafficking and the innate immune response play a complex and important role in mediating the pulmonary inflammatory response and dysfunction during renal IRI.

3.1.2. Lung Apoptosis. Though not traditionally associated with a proinflammatory response, pulmonary apoptosis plays a critical role in the genomic and phenotypic response to ischemic AKI. Apoptosis of pulmonary epithelial and endothelial cells along with delayed leukocyte apoptosis occurs during ALI [50-52]. Pulmonary apoptosis disrupts tethering forces involved in cell-to-cell and cell-toextracellular matrix interactions, leading to a loss of endothelial barrier function and increased vascular permeability 


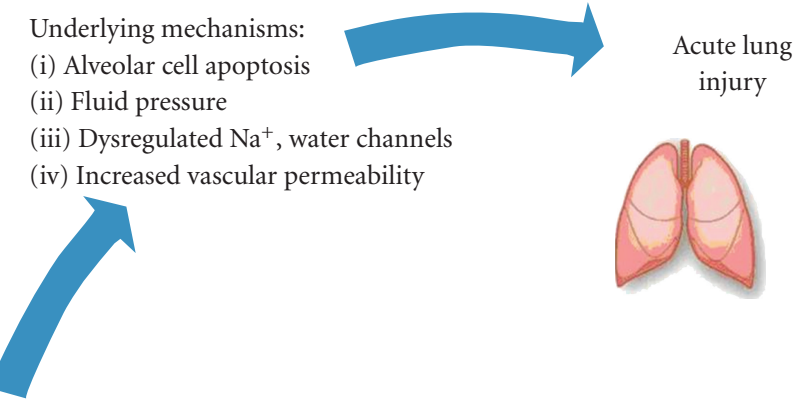

\begin{abstract}
Mediators:
(i) Oxidative stress

(ii) Cytokines/chemokines

(iii) Activated leukocytes

(iv) Uremic toxins
\end{abstract}
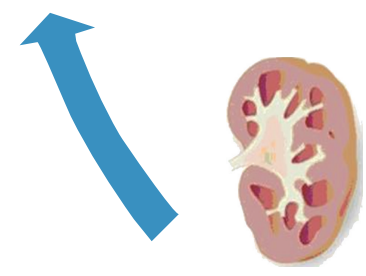

Acute kidney injury

\section{Pathological axis \\ between AKI and ALI}

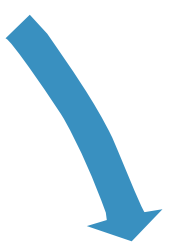

Mediators:

(i) Hypoxia

(ii) Hypercapnia

(iii) PEEP

(iv) Biotrauma

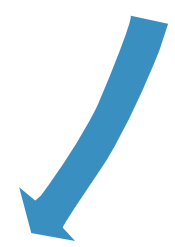

Underlying mechanisms:

(i) Decreased renal perfusion

(ii) Blood-gas disturbances

(iii) Inflammation/apoptosis

FIgure 2: Pathological Axis between AKI and ALI. AKI induces pathophysiologic effects on the lung via cellular and soluble mediators. ALI, in turn, exacerbates kidney dysfunction through metabolic and biochemical derangements.

[53]. In a rodent model, our laboratory has characterized caspase-dependent pulmonary apoptosis which is abrogated by the administration of a broad-spectrum caspase inhibitor. Not only did caspase inhibition attenuate the pulmonary functional injury, but the proapoptotic response to ischemic AKI appears to be mediated via a TNF receptor-1 (TNF-R-1) dependent pathway [54].

Our research has also demonstrated T-cell-dependent pulmonary apoptosis during ischemic AKI. Along with identification of activated $\mathrm{T}$ cells trafficking to the lungs during ischemic AKI, we have found that pulmonary apoptosis was subsequently attenuated in T-cell-deficient animals [33]. Further pursuit of the specific pathway for T cell and TNFR1dependent pulmonary apoptosis remains an active focus of future investigation in our research laboratory.

3.2. Kidney Crosstalk with Other Distant Organs. Cardiovascular collapse is one of the most common causes of death in the setting of AKI, yet the mechanisms involved are not entirely understood [55]. Kelly has demonstrated left ventricular dilation, increased left ventricular end diastolic and end systolic diameter, increased relaxation time, and decreased fractional shortening in an experimental model of ischemic AKI [56]. Mechanisms of cardiac injury during ischemic AKI include cardiac myocyte apoptosis and neutrophil infiltration, which have been attributed to increased cardiac and systemic TNF $\alpha$, IL-1, and ICAM-1 expression $[56,57]$. When kidney ischemic time is decreased, cardiac
IL-1 and ICAM expression, along with myocyte necrosis, decreased correspondingly [56].

Effects of ischemic AKI on the central nervous system (CNS) are evident clinically when mental status changes develop. Of note, renal replacement therapy fails to fully correct this CNS manifestation of renal failure [58]. Uremic toxins certainly contribute to many symptoms of encephalopathy; however, cellular and soluble inflammatory mediators have also been implicated. Preclinical data identified an increase of $\mathrm{KC}, \mathrm{G}-\mathrm{CSF}$, and glial fibrillary acidic protein (GFAP) in the cerebral cortex and hippocampus of the brain, which may serve to recruit neutrophils to sites of neuronal damage [59]. This may result from either increased local neuronal production or an alteration of the blood-brain barrier; further research is required to delineate the source. A cell-mediated proinflammatory response has also been identified with activation of microglial cells (brain macrophages) during ischemic AKI [59].

Ischemic AKI is also implicated in oxidative stress, inflammation, apoptosis, and tissue damage in hepatocytes. Hepatic stellate cells (HSCs) regulate leukocyte trafficking and the secretion of chemokines such as IL-8, and crosstalk between HSCs likely occurs via a c-Jun N-terminal kinase pathway [60]. Oxidative stress during ischemic AKI causes hepatic malondialdehyde, an index of lipid peroxidation, to increase while total glutathione, an antioxidant, decreases [61]. Proinflammatory cytokine $\mathrm{TNF} \alpha$ expression and hepatic cellular apoptosis is also evident during ischemic AKI [61]. 
Previous investigators and clinicians have labeled the gut as the "motor" of MOF because of its ability to amplify the systemic SIRS response in the setting of shock and gut hypoperfusion [62-64]. These mechanisms include increased intestinal permeability, interactions between host and bacterial pathogens, and propagation of toxins to distant organs via the lymphatic system $[63,65]$ and could potentially play a role during ischemic AKI. Clinical studies have long demonstrated the increased secretion of potassium by the colon and rectum in response to AKI [66], and recent literature linked channel-inducing factor, a potassium channel regulator found in both the kidney and colon, to ischemic AKI $[67,68]$. This may provide explanation for why hyperkalemia does not universally occur during AKI. The role of the gut in response to organ crosstalk during ischemic AKI remains a fascinating topic of future investigation.

\section{Conclusion}

AKI is a frequent complication amongst hospitalized patients, with grave implications in the setting of MOF. Ischemic AKI initiates a cascade of proinflammatory pathways, and through the release of soluble mediators and activation of the host innate and adaptive immune systems, it facilitates organ crosstalk and remote organ injury. As our understanding of the postischemic kidney's role in mediating organ crosstalk continues to evolve, further laboratory investigation into the remote organ response to this devastating injury may reveal potential future therapeutic targets.

\section{References}

[1] E. M. Levy, C. M. Viscoli, and R. I. Horwitz, "The effect of acute renal failure on mortality: a cohort analysis," Journal of American Medical Association, vol. 275, no. 19, pp. 1489-1494, 1996.

[2] P. G. H. Metnitz, C. G. Krenn, H. Steltzer et al., "Effect of acute renal failure requiring renal replacement therapy on outcome in critically ill patients," Critical Care Medicine, vol. 30, no. 9, pp. 2051-2058, 2002.

[3] H. E. Bass, D. Greenberg, E. Singer, and M. A. Miller, "Pulmonary changes in uremia," Journal of American Medical Association, vol. 148, pp. 724-726, 1952.

[4] M. R. Back, M. Bandyk, M. Bradner et al., "Critical analysis of outcome determinants affecting repair of intact aneurysms involving the visceral aorta," Annals of Vascular Surgery, vol. 19, no. 5, pp. 648-656, 2005.

[5] V. S. Kashyap, R. P. Cambria, J. K. Davison, and G. J. L'Italien, "Renal failure after thoracoabdominal aortic surgery," Journal of Vascular Surgery, vol. 26, no. 6, pp. 949-955, 1997.

[6] L. G. Svensson, E. S. Crawford, K. R. Hess, J. S. Coselli, and H. J. Safi, "Experience with 1509 patients undergoing thoracoabdominal aortic operations," Journal of Vascular Surgery, vol. 17, no. 2, pp. 357-368, 1993.

[7] J. J. Friedewald and H. Rabb, "Inflammatory cells in ischemic acute renal failure," Kidney International, vol. 66, no. 2, pp. 486-491, 2004.

[8] M. Brezis and S. Rosen, "Mechanisms of disease: hypoxia of the renal medulla-its implications for disease," New England Journal of Medicine, vol. 332, no. 10, pp. 647-655, 1995.
[9] Z. Wang, H. Rabb, T. Craig, C. Burnham, G. E. Shull, and M. Soleimani, "Ischemic-reperfusion injury in the kidney: overexpression of colonic $\mathrm{H}^{+}-\mathrm{K}^{+}$-ATPase and suppression of NHE-3," Kidney International, vol. 51, no. 4, pp. 1106-1115, 1997.

[10] J. V. Bonventre and A. Zuk, "Ischemic acute renal failure: an inflammatory disease?" Kidney International, vol. 66, no. 2, pp. 480-485, 2004.

[11] H. R. Jang, G. J. Ko, B. A. Wasowska, and H. Rabb, "The interaction between ischemia-reperfusion and immune responses in the kidney," Journal of Molecular Medicine, vol. 87, no. 9, pp. 859-864, 2009.

[12] H. T. Hassoun, D. N. Grigoryev, M. L. Lie et al., "Ischemic acute kidney injury induces a distant organ functional and genomic response distinguishable from bilateral nephrectomy," American Journal of Physiology, vol. 293, no. 1, pp. F30F40, 2007.

[13] J. Deng, X. Hu, P. S. T. Yuen, and R. A. Star, “ $\alpha$ melanocyte-stimulating hormone inhibits lung injury after renal ischemia/reperfusion," American Journal of Respiratory and Critical Care Medicine, vol. 169, no. 6, pp. 749-756, 2004.

[14] H. Yoshidome, A. Kato, M. J. Edwards, and A. B. Lentsch, "Interleukin-10 inhibits pulmonary NF- $\kappa$ B activation and lung injury induced by hepatic ischemia-reperfusion," American Journal of Physiology, vol. 277, no. 5, pp. L919-L923, 1999.

[15] K. J. Kelly, W. W. Williams Jr., R. B. Colvin et al., "Intercellular adhesion molecule-1-deficient mice are protected against ischemic renal injury," Journal of Clinical Investigation, vol. 97, no. 4, pp. 1056-1063, 1996.

[16] K. K. Donnahoo, X. Meng, A. Ayala, M. P. Cain, A. H. Harken, and D. R. Meldrum, "Early kidney TNF- $\alpha$ expression mediates neutrophil infiltration and injury after renal ischemiareperfusion," American Journal of Physiology, vol. 277, no. 3, pp. R922-R929, 1999.

[17] H. Chiao, Y. Kohda, P. McLeroy, L. Craig, I. Housini, and R. A. Star, " $\alpha$-melanocyte-stimulating hormone protects against renal injury after ischemia in mice and rats," Journal of Clinical Investigation, vol. 99, no. 6, pp. 1165-1172, 1997.

[18] T. Chakraborti, A. Mandal, M. Mandal, S. Das, and S. Chakraborti, "Complement activation in heart diseases: role of oxidants," Cellular Signalling, vol. 12, no. 9-10, pp. 607-617, 2000.

[19] H. Rabb, "The T cell as a bridge between innate and adaptive immune systems: implications for the kidney," Kidney International, vol. 61, no. 6, pp. 1935-1946, 2002.

[20] F. Arslan, B. Keogh, P. McGuirk, and A. E. Parker, "TLR2 and TLR4 in ischemia reperfusion injury," Mediators of Inflammation, vol. 2010, Article ID 704202, 8 pages, 2010.

[21] J. C. Leemans, G. Stokman, N. Claessen et al., "Renalassociated TLR2 mediates ischemia/reperfusion injury in the kidney," Journal of Clinical Investigation, vol. 115, no. 10, pp. 2894-2903, 2005.

[22] W. P. Pulskens, G. J. Teske, L. M. Butter et al., “Toll-like receptor-4 coordinates the innate immune response of the kidney to renal ischemia/reperfusion injury," PLOS ONE, vol. 3, no. 10, article e3596, 2008.

[23] S. Wang, C. Schmaderer, E. Kiss et al., "Recipient toll-like receptors contribute to chronic graft dysfunction by both MyD88- and TRIF-dependent signaling," Disease Models and Mechanisms, vol. 3, no. 1-2, pp. 92-103, 2010.

[24] Y. Horie, R. P. Chervenak, R. Wolf et al., "Lymphocytes mediate TNF- $\alpha$-induced endothelial cell adhesion molecule expression: studies on SCID and RAG-1 mutant mice," Journal of Immunology, vol. 159, no. 10, pp. 5053-5062, 1997. 
[25] Y. Okada, X. J. Zuo, A. M. Marchevsky et al., "Antithrombin III treatment improves parameters of acute inflammation in a highly histoincompatible model of rat lung allograft rejection," Transplantation, vol. 67, no. 4, pp. 526-528, 1999.

[26] R. N. Puglisi, L. Strande, M. Santos, G. Schulte, C. W. Hewitt, and T. V. Whalen, "Beneficial effects of cyclosporine and rapamycin in small bowel ischemic injury," Journal of Surgical Research, vol. 65, no. 2, pp. 115-118, 1996.

[27] J. Sharkey and S. P. Butcher, "Immunophilins mediate the neuroprotective effects of FK506 in focal cerebral ischaemia," Nature, vol. 371, no. 6495, pp. 336-339, 1994.

[28] N. Varda-Bloom, J. Leor, D. G. Ohad et al., "Cytotoxic T lymphocytes are activated following myocardial infarction and can recognize and kill healthy myocytes in vitro," Journal of Molecular and Cellular Cardiology, vol. 32, no. 12, pp. 21412149, 2000.

[29] P. Boros and J. S. Bromberg, "New cellular and molecular immune pathways in ischemia/reperfusion injury," American Journal of Transplantation, vol. 6, no. 4, pp. 652-658, 2006.

[30] H. Rabb, F. Daniels, M. O’Donnell et al., "Pathophysiological role of $\mathrm{T}$ lymphocytes in renal ischemia-reperfusion injury in mice," American Journal of Physiology, vol. 279, no. 3, pp. F525-F531, 2000.

[31] M. J. Burne, F. Daniels, A. El Ghandour et al., "Identification of the CD4(+) T cell as a major pathogenic factor in ischemic acute renal failure," Journal of Clinical Investigation, vol. 108, no. 9, pp. 1283-1290, 2001.

[32] N. Yokota, M. Burne-Taney, L. Racusen, and H. Rabb, "Contrasting roles for STAT4 and STAT6 signal transduction pathways in murine renal ischemia-reperfusion injury," American Journal of Physiology, vol. 285, no. 2, pp. F319-F325, 2003.

[33] M. L. Lie, R. J. Santora, H. Rabb, and H. T. Hassoun, "Distant organ T Cell trafficking and activation during Ischemic Acute Kidney Injury (AKI)," Journal of Surgical Research, vol. 158, p. 291, 2010.

[34] D. B. Ascon, S. Lopez-Briones, M. Liu et al., "Phenotypic and functional characterization of kidney-infiltrating lymphocytes in renal ischemia reperfusion injury," Journal of Immunology, vol. 177 , no. 5, pp. 3380-3387, 2006.

[35] K. Hochegger, T. Schätz, P. Eller et al., "Role of $\alpha / \beta$ and $\gamma / \delta$ T cells in renal ischemia-reperfusion injury," American Journal of Physiology, vol. 293, no. 3, pp. F741-F747, 2007.

[36] S. R. Satpute, J. M. Park, H. R. Jang et al., "The role for T cell repertoire/antigen-specific interactions in experimental kidney ischemia reperfusion injury," Journal of Immunology, vol. 183, no. 2, pp. 984-992, 2009.

[37] M. Ascon, D. B. Ascon, M. Liu et al., "Renal ischemiareperfusion leads to long term infiltration of activated and effector-memory T lymphocytes," Kidney International, vol. 75, no. 5, pp. 526-535, 2009.

[38] M. J. Burne-Taney, M. Liu, D. Ascon, R. R. Molls, L. Racusen, and H. Rabb, "Transfer of lymphocytes from mice with renal ischemia can induce albuminuria in naive mice: a possible mechanism linking early injury and progressive renal disease?" American Journal of Physiology, vol. 291, no. 5, pp. F981-F986, 2006.

[39] M. J. Burne-Taney, D. B. Ascon, F. Daniels, L. Racusen, W. Baldwin, and H. Rabb, "B cell deficiency confers protection from renal ischemia reperfusion injury," Journal of Immunology, vol. 171, no. 6, pp. 3210-3215, 2003.

[40] R. L. Mehta, M. T. Pascual, C. G. Gruta, S. Zhuang, and G. M. Chertow, "Refining predictive models in critically ill patients with acute renal failure," Journal of the American Society of Nephrology, vol. 13, no. 5, pp. 1350-1357, 2002.
[41] Z. Ricci and C. Ronco, "Pulmonary/renal interaction," Current Opinion in Critical Care, vol. 16, no. 1, pp. 13-18, 2010.

[42] G. R. Bernard, A. Artigas, K. L. Brigham et al., "The AmericanEuropean Consensus Conference on ARDS: definitions, mechanisms, relevant outcomes, and clinical trial coordination," American Journal of Respiratory and Critical Care Medicine, vol. 149, no. 3, pp. 818-824, 1994.

[43] A. A. Kramer, G. Postler, K. F. Salhab, C. Mendez, L. C. Carey, and H. Rabb, "Renal ischemia/reperfusion leads to macrophage-mediated increase in pulmonary vascular permeability," Kidney International, vol. 55, no. 6, pp. 2362-2367, 1999.

[44] H. Rabb, Z. Wang, T. Nemoto, J. Hotchkiss, N. Yokota, and M. Soleimani, "Acute renal failure leads to dysregulation of lung salt and water channels," Kidney International, vol. 63, no. 2, pp. 600-606, 2003.

[45] C. L. Klein, T. S. Hoke, W. F. Fang, C. J. Altmann, I. S. Douglas, and S. Faubel, "Interleukin-6 mediates lung injury following ischemic acute kidney injury or bilateral nephrectomy," Kidney International, vol. 74, no. 7, pp. 901-909, 2008.

[46] T. S. Hoke, I. S. Douglas, C. L. Klein et al., "Acute renal failure after bilateral nephrectomy is associated with cytokinemediated pulmonary injury," Journal of the American Society of Nephrology, vol. 18, no. 1, pp. 155-164, 2007.

[47] D. J. Kim, S. H. Park, M. R. Sheen et al., "Comparison of experimental lung injury from acute renal failure with injury due to sepsis," Respiration, vol. 73, no. 6, pp. 815-824, 2006.

[48] D. N. Grigoryev, M. Liu, H. T. Hassoun, C. Cheadle, K. C. Barnes, and H. Rabb, "The local and systemic inflammatory transcriptome after acute kidney injury," Journal of the American Society of Nephrology, vol. 19, no. 3, pp. 547-558, 2008.

[49] A. Zarbock, M. Schmolke, T. Spieker, K. Jurk, H. Van Aken, and K. Singbartl, "Acute uremia but not renal inflammation attenuates aseptic acute lung injury: a critical role for uremic neutrophils," Journal of the American Society of Nephrology, vol. 17, no. 11, pp. 3124-3131, 2006.

[50] G. Matute-Bello, W. C. Liles, F. Radella II et al., "Neutrophil apoptosis in the acute respiratory distress syndrome," American Journal of Respiratory and Critical Care Medicine, vol. 156, no. 6, pp. 1969-1977, 1997.

[51] G. Matute-Bello, W. C. Liles, K. P. Steinberg et al., "Soluble Fas ligand induces epithelial cell apoptosis in humans with acute lung injury (ARDS)," Journal of Immunology, vol. 163, no. 4, pp. 2217-2225, 1999.

[52] A. Q. Rafi, A. Zeytun, M. J. Bradley et al., "Evidence for the involvement of Fas ligand and perforin in the induction of vascular leak syndrome," Journal of Immunology, vol. 161, no. 6, pp. 3077-3086, 1998.

[53] S. M. Dudek and J. G. N. Garcia, "Cytoskeletal regulation of pulmonary vascular permeability," Journal of Applied Physiology, vol. 91, no. 4, pp. 1487-1500, 2001.

[54] H. T. Hassoun, M. L. Lie, D. N. Grigoryev, M. Liu, R. M. Tuder, and H. Rabb, "Kidney ischemia-reperfusion injury induces caspase-dependent pulmonary apoptosis," American Journal of Physiology, vol. 297, no. 1, pp. F125-F137, 2009.

[55] P. Blake, Y. Hasegawa, M. C. Khosla, F. Fouad-Tarazi, N. Sakura, and E. P. Paganini, "Isolation of "myocardial depressant factor(s)" from the ultrafiltrate of heart failure patients with acute renal failure," ASAIO Journal, vol. 42, no. 5, pp. M911-M915, 1996.

[56] K. J. Kelly, "Distant effects of experimental renal ischemia/ reperfusion injury," Journal of the American Society of Nephrology, vol. 14, no. 6, pp. 1549-1558, 2003. 
[57] D. Bryant, L. Becker, J. Richardson et al., "Cardiac failure in transgenic mice with myocardial expression of tumor necrosis factor- $\alpha$, Circulation, vol. 97, no. 14, pp. 1375-1381, 1998.

[58] R. Brouns and P. P. De Deyn, "Neurological complications in renal failure: a review," Clinical Neurology and Neurosurgery, vol. 107, no. 1, pp. 1-16, 2004.

[59] M. Liu, Y. Liang, S. Chigurupati et al., "Acute kidney injury leads to inflammation and functional changes in the brain," Journal of the American Society of Nephrology, vol. 19, no. 7, pp. 1360-1370, 2008.

[60] R. F. Schwabe, B. Schnabl, Y. O. Kweon, and D. A. Brenner, "CD40 activates NF- $\kappa$ B and c-Jun N-terminal kinase and enhances chemokine secretion on activated human hepatic stellate cells," Journal of Immunology, vol. 166, no. 11, pp. 6812-6819, 2001.

[61] F. Golab, M. Kadkhodaee, M. Zahmatkesh et al., "Ischemic and non-ischemic acute kidney injury cause hepatic damage," Kidney International, vol. 75, no. 8, pp. 783-792, 2009.

[62] C. J. Carrico, J. L. Meakins, J. C. Marshall, D. Fry, and R. V. Maier, "Multiple-organ-failure syndrome," Archives of Surgery, vol. 121, no. 2, pp. 196-208, 1986.

[63] J. A. Clark and C. M. Coopersmith, "Intestinal crosstalk: a new paradigm for understanding the gut as the "motor" of critical illness," Shock, vol. 28, no. 4, pp. 384-393, 2007.

[64] H. T. Hassoun, B. C. Kone, D. W. Mercer, F. G. Moody, N. W. Weisbrodt, and F. A. Moore, "Post-injury multiple organ failure: the role of the gut," Shock, vol. 15, no. 1, pp. 1-10, 2001.

[65] M. P. Fink and R. L. Delude, "Epithelial barrier dysfunction: a unifying theme to explain the pathogenesis of multiple organ dysfunction at the cellular level," Critical Care Clinics, vol. 21, no. 2, pp. 177-196, 2005.

[66] R. S. Martin, S. Panese, M. Virginillo et al., "Increased secretion of potassium in the rectum of humans with chronic renal failure," American Journal of Kidney Diseases, vol. 8, no. 2, pp. 105-110, 1986.

[67] D. Gimelreich, M. M. Popovtzer, H. Wald, G. Pizov, Y. Berlatzky, and D. Rubinger, "Regulation of ROMK and channel-inducing factor (CHIF) in acute renal failure due to ischemic reperfusion injury," Kidney International, vol. 59, no. 5, pp. 1812-1820, 2001.

[68] H. Rabb, Z. Wang, G. Postler, and M. Soleimani, "Possible molecular basis for changes in potassium handling in acute renal failure," American Journal of Kidney Diseases, vol. 35, no. 5, pp. 871-877, 2000. 


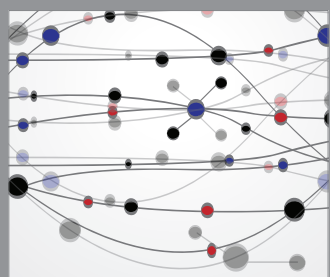

The Scientific World Journal
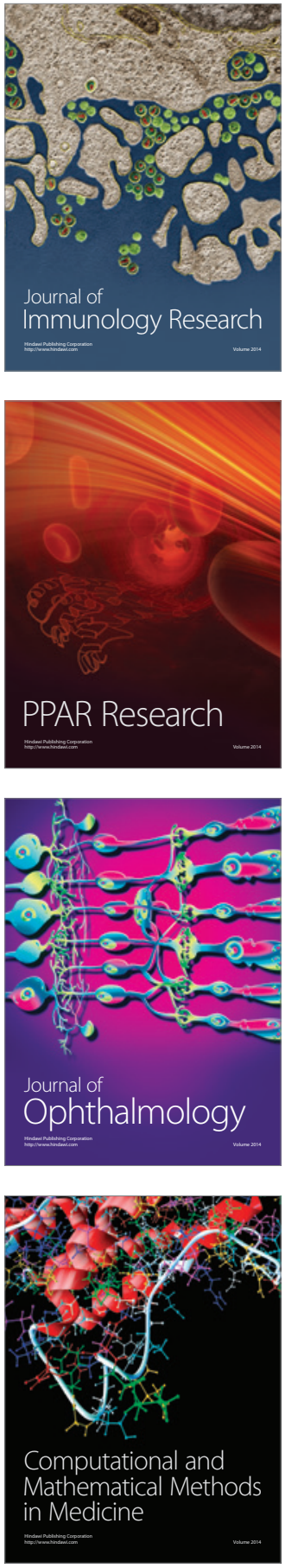

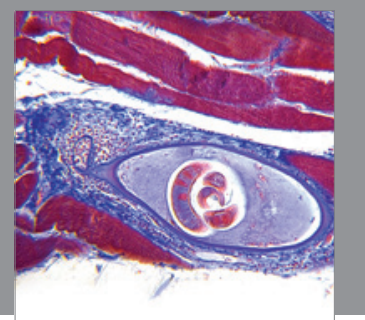

Gastroenterology

Research and Practice
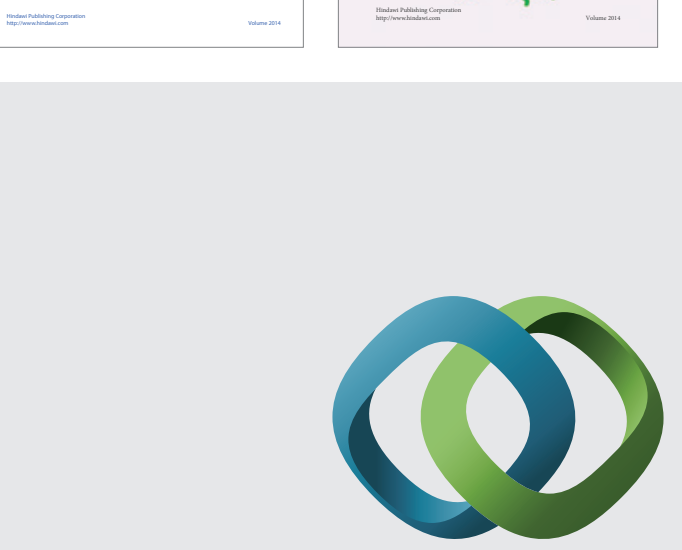

\section{Hindawi}

Submit your manuscripts at

http://www.hindawi.com
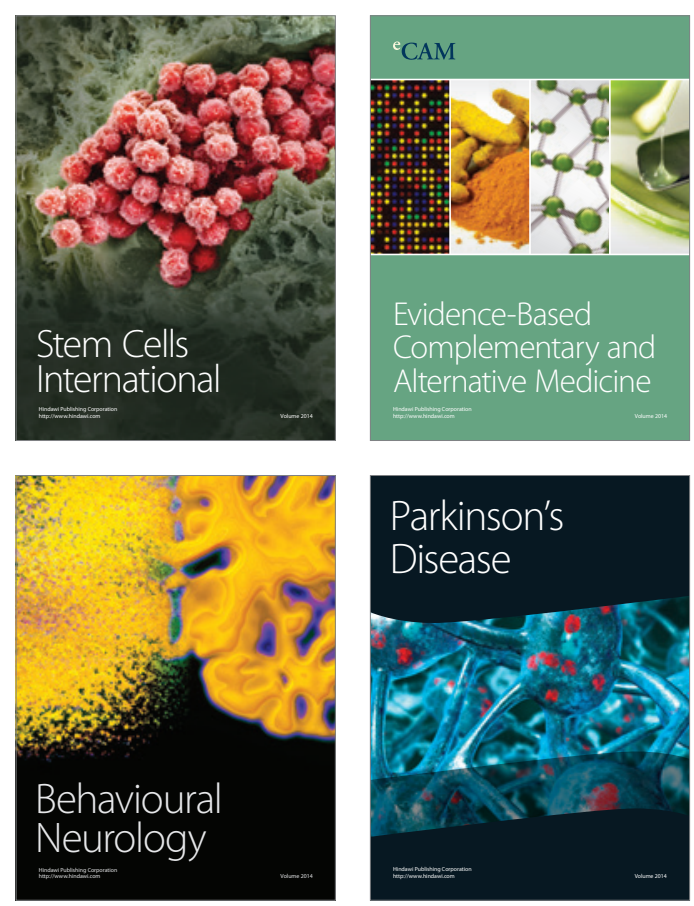

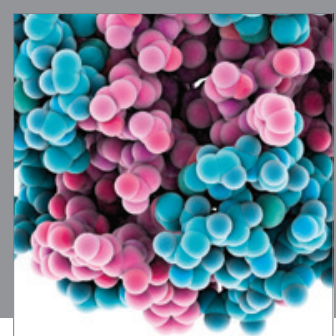

Journal of
Diabetes Research

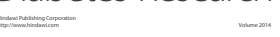

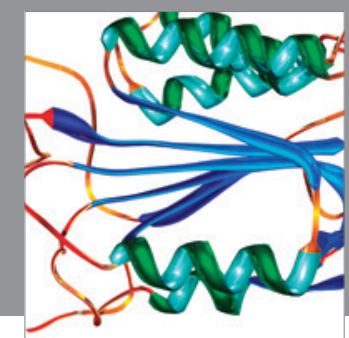

Disease Markers
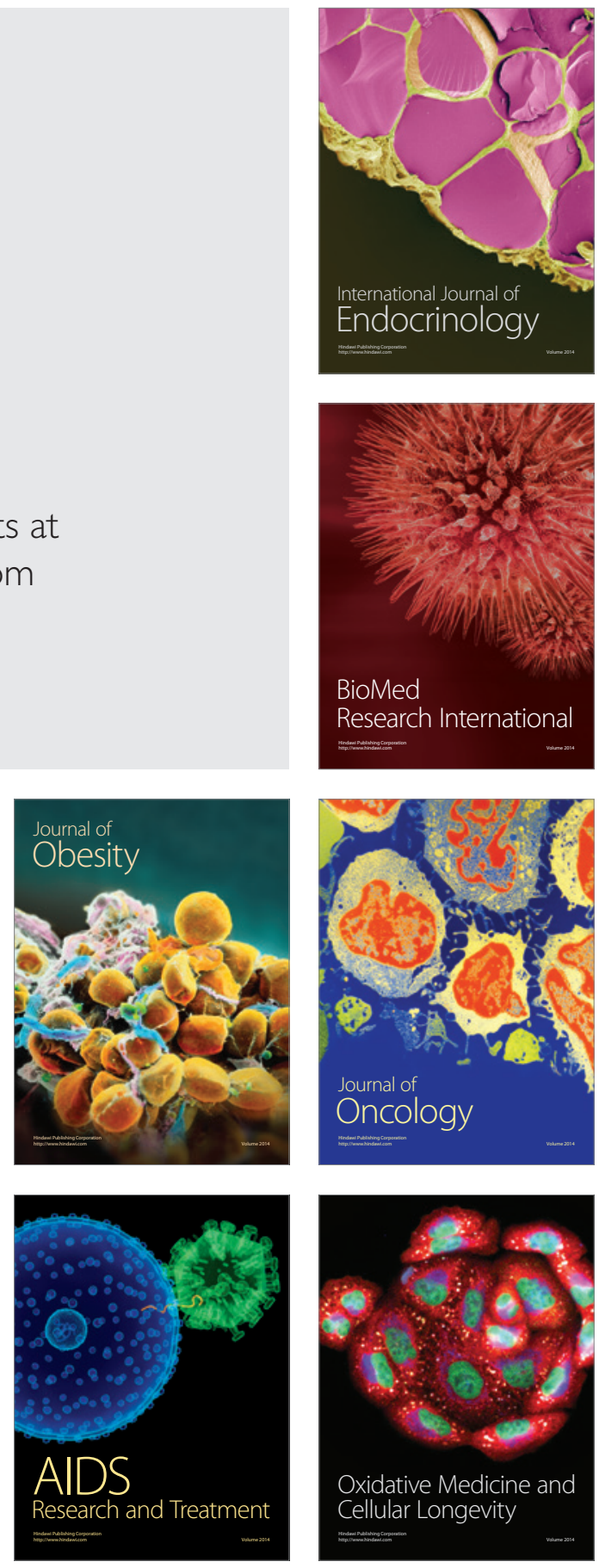